CERN-TH-6260/91

\title{
SUPERGRAVITY DARK MATTER
}

\author{
John Ellis and Leszek Roszkowski ${ }^{\star}$ \\ Theoretical Physics Division, CERN \\ CH - 1211 Geneva 23
}

\section{ERRATUM}

Due to a printing error, the enclosed Figs. 4 (c), (d) were missing from the end of this report.

CERN-TH-6260/91

September 1991

* Address after October 1, 1991: Physics Department, University of Michigan, Ann Arbor, MI 48109, USA; bitnet address: leszek@umiphys. 


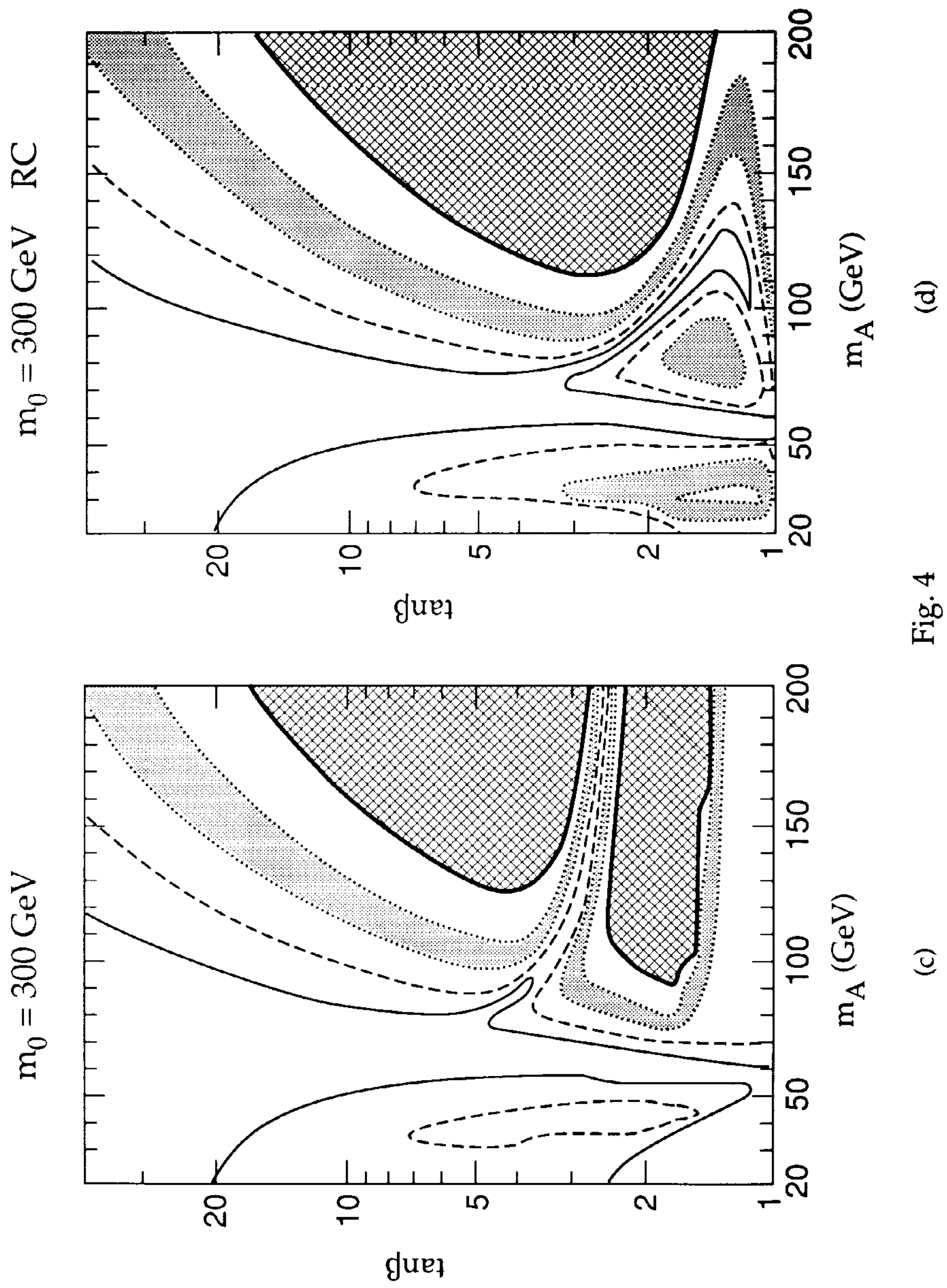